\title{
NONINTRUSIVE DIAGNOSTICS OF HIGH-TEMPERATURE MEDIA AND OBJECTS AT MOTOR TESTING
}

\author{
V. V. Mironov, N. N. Volkov, L. I. Volkova, \\ V.I. Kondratenko, V. A. Popov, \\ L. B. Treshchalin, and S. M. Tsatsuev
}

Measuring complex allowing diagnostics of heated-object thermal fields with high resolution was developed. Numerical model was also developed for nozzle tests in vacuum chamber. The model allows simulating the main specific features of the tests. A comparison is made between experimental and calculated results.

\section{INTRODUCTION. MEASURING COMPLEX}

Conventional means used for temperature and heat flux measurements (thermocouples, thermoresistors, thermosensors, calorimeters) despite possessing high precision and stability of characteristics, exhibit a number of drawbacks (sluggishness, limited number of metering points, lifetime and temperature limits in corrosive environments, etc.). A new metering complex was developed in Keldysh Research Center allowing fine-resolution diagnostic of thermal fields and heated objects (Fig. 1) to enhance engine thermal diagnostics during testing and to expand the amount of data collected (high-temperature objects, first of all).

Nonintrusive measuring method is realized by applying an optico-electronic system. Its main technical characteristics are listed below:

- spectral sensitivity $-0,9-1 \mu \mathrm{m}$;

- sensitive element - $\mathrm{CCD}^{*}$ matrix;

- number of operating elements of CCD matrix $-740 \times 580$;

- exposition time - from $45 \mu \mathrm{s}$ to $40 \mathrm{~s}$;

- temperature metering interval $-650-1700{ }^{\circ} \mathrm{C}$;

${ }^{*}$ CCD - charge-coupled device. 


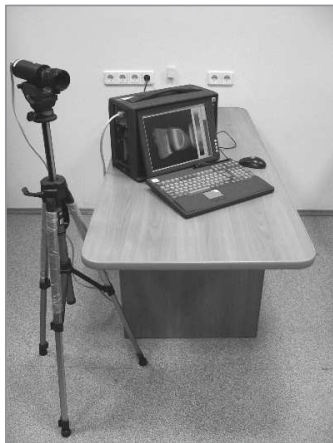

(a)

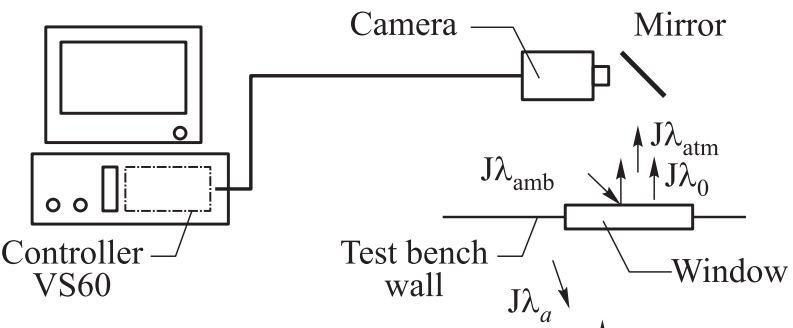

Additional mirror

(b)

Figure 1 Measuring complex for nonintrusive diagnostic of high-temperature environments: $(a)$ overall view; and (b) functional scheme

- temperature metering interval with additional neutral filter — from 900 to $4000{ }^{\circ} \mathrm{C}$;

- basic error $-3{ }^{\circ} \mathrm{C}$ below $1500{ }^{\circ} \mathrm{C}$ and $\pm 1 \%$ above; and

- framing frequency - up to $25 \mathrm{~s}^{-1}$.

Infrared (IR) camera is assigned to transform a signal coming from a heated surface which is interpreted by sensitive elements proportionally. The camera operates in the following way. A major part of heat radiation from the examined object passes through IR mirror and a remaining part (in the band of 0,9-1.1micrometer spectrum) is reflected to camera. Due to filters installed on the object, the CCD matrix receives radiation only in a narrow spectral band. Each CCD element forms a signal which is proportional to the energy of received radiation.

This signal is digitized and sent to a controller which controls camera functioning and provides signal transfer from camera to computer. A computer program processes data and visualizes the thermal image of tested object. Calibration constants for calculating temperatures based on the digital data are determined using thermal lamp or a black-body model.

Thermovisor software allows image output on the computer screen with a frequency up to 25 frames/s. Images can be recorded on a hard disc frame by frame with any operator-predetermined rate which is limited only by computer productivity. Also, the software allows processing the saved images.

In conjunction with other means of diagnostics, the metering complex is used for diagnostics of uncooled nozzle state (Figs. 2 to 4 ). 


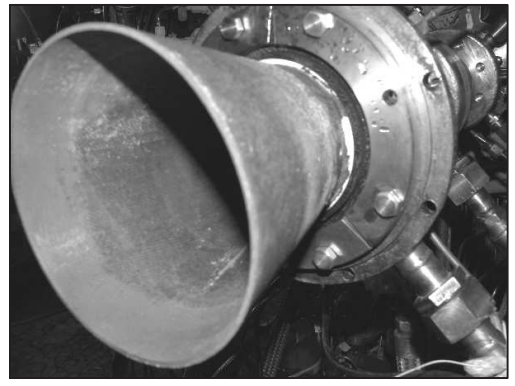

Figure 2 Uncooled nozzle model

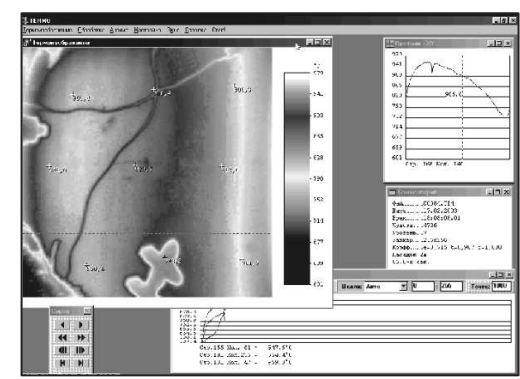

Figure 3 Heated nozzle thermoimage

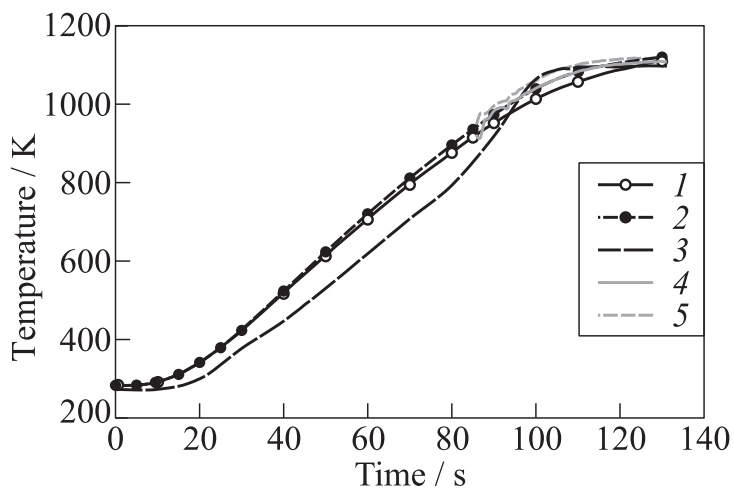

Figure 4 Nozzle external wall temperature measured by thermocouple, nonintrusive method and calculated using numerical method: 1 - one-dimensional calculation; $2-$ two-dimensional calculation; 3 - thermocouple No. 6 ; 4 - thermosensor No. 1 ; and 5 - thermosensor No. 2

\section{EXPERIMENTAL INVESTIGATION}

Nozzle tests, especially when high expansion degrees are modeled, demand the use of a vacuum chamber docked with diffuser. The vacuum chamber is aimed to meet two requirements. First, it should embrace the nozzle as a whole (Fig. 5), to prevent contact between the nozzle external surface and air (thus preventing intense oxidation of nozzle material as well as its intense ablation and destruction). Second, it should be docked to a cooled exhaust tube, long enough to support a required pressure drop as a result of jet ejection and thus to ensure continuous outflow of combustion products even at high expansion degrees (when the pressure at the nozzle section exit is near 0.06-0.08 bar). As for the internal 


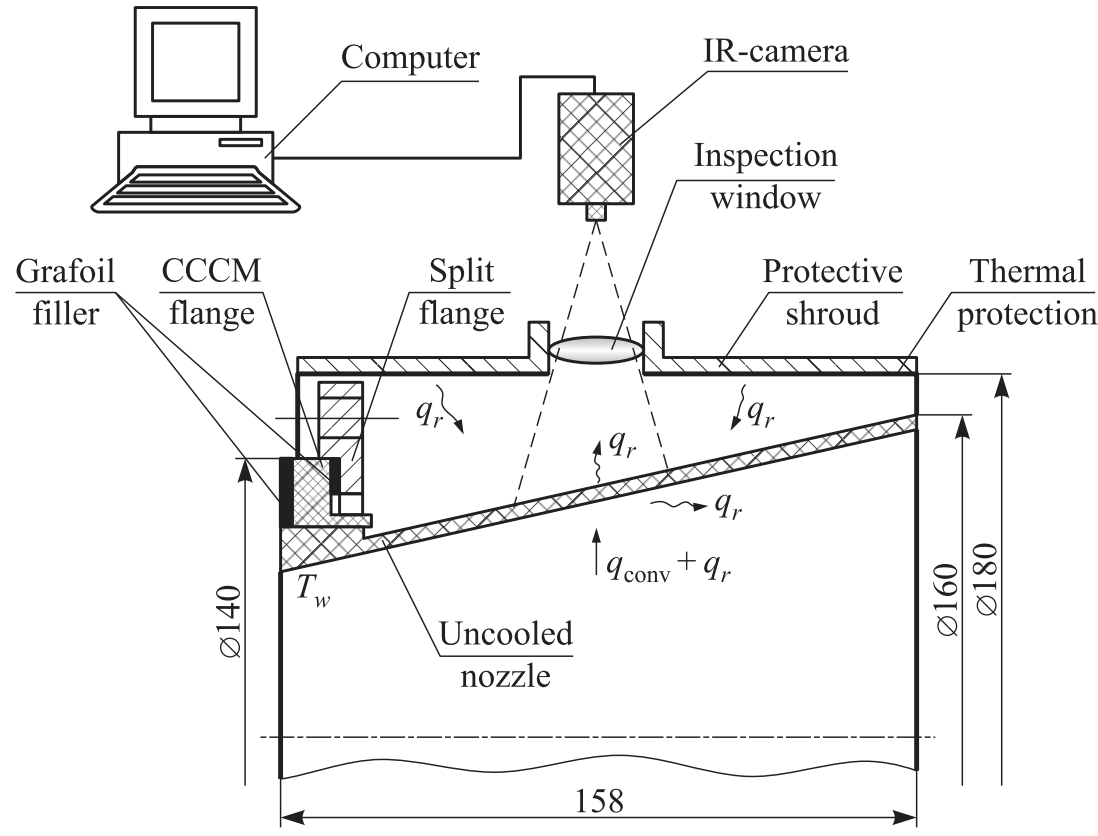

Figure 5 Schematic of extension arrangement in the vacuum chamber. Dimensions are in millimeters

surface of the vacuum chamber, it should be thermoprotected to support the demanded conditions for nozzle temperature.

The thermoprotection surface is heated during the experiment which causes the radiant heat flux to nozzle. Therefore, it was necessary to create a technique, allowing right examination of test results for adequate target setting and results analysis.

Oxygen-hydrogen liquid rocket engine (LRE) in vacuum chamber with diffuser test conditions was reconstituted to develop the computational model. The nozzle was placed into the vacuum chamber with inner thermoprotection made of coke material (see Fig. 5).

\section{Numerical Model of Thermal State}

Thermal state of such a system is described by two equations of thermal conductivity (along the vacuum chamber and nozzle surfaces) subject to proper boundary conditions: 
- for vacuum chamber:

$$
c_{p} \rho_{p} \frac{\partial T}{\partial t}=\frac{1}{r} \frac{\partial}{\partial r}\left(r \lambda_{\cdot p} \frac{\partial T}{\partial r}\right)+c_{g} G \frac{\partial T}{\partial r}+\frac{d \rho_{m}}{s t}\left[\frac{Q_{s}}{\chi_{g}}+c_{g} T\left(1-\frac{\rho_{0}}{\rho_{g}}\right)\right] ;
$$

- for nozzle:

$$
c_{e} \rho_{e} \frac{\partial T}{\partial t}=\frac{1}{r} \frac{\partial}{\partial r}\left(r \lambda_{e} \frac{\partial T}{\partial r}\right)+c_{g 1} G \frac{\partial T}{\partial r}+\frac{d \rho_{e}}{d t}\left[\frac{Q_{s 1}}{\chi_{g 1}}+c_{g 1} T\left(1-\frac{\rho_{01}}{\rho_{g 1}}\right)\right] .
$$

Source and convection terms are kept in the second equation to make a provision of taking into account the thermochemical processes inside the nozzle.

The effect of the thermoprotection bunch on thermodestruction process is described by the overall reaction:

$$
-\frac{d \rho_{m}}{d t}=k_{0} \chi \rho_{0}\left(\beta-\frac{\varepsilon}{\chi}\right) e^{-E_{m} /(R T)} .
$$

Therefore,

$$
\begin{aligned}
\varepsilon & =\int_{0}^{t} \chi k_{0}\left(\beta-\frac{\varepsilon}{\chi}\right) e^{-E_{m} /(R T)} d t \\
G & =-\frac{1}{r} \int_{r_{s 1}}^{r_{s 1}+\delta} r\left(1-\frac{\rho_{g}}{\rho_{0}}\right) \frac{d \rho_{m}}{d t} d r .
\end{aligned}
$$

Here, $\beta$ is the relative bunch content in the initial material; $\chi=(1-k) \beta$; $k$ is the coke number; $\varepsilon$ is the material porosity; $E_{0}$ is the activation energy of thermodestruction process; $k_{m}$ is the preexponential factor for this process; and $G$ is the mass flow rate of destruction products.

Initial condition: $T\left(r, t_{0}\right)=T_{0}$.

Boundary conditions:

- on the nozzle internal surface

$$
-\left.\lambda \frac{d T}{d r}\right|_{r_{s 1}}=\frac{\alpha}{c_{p}}\left(J_{e}-J_{w}\right)-\dot{m}_{w} H_{s}-\varphi \varepsilon \sigma\left(\frac{T_{w}}{100}\right)^{4}+Q_{\text {add }}
$$

- on the nozzle external surface

$$
\left.\lambda \frac{d T}{d r}\right|_{r_{s 12}}=-\varepsilon_{\mathrm{ad} j} \sigma \varphi_{12}\left[\left(\frac{T_{w 12}}{100}\right)^{4}-\left(\frac{T_{w 21}}{100}\right)^{4}\right]
$$


- on the vacuum-chamber internal surface

$$
-\left.\lambda \frac{d T}{d r}\right|_{r_{s 21}}=-\varepsilon_{\mathrm{ad} j} \sigma \varphi_{21}\left[\left(\frac{T_{w 12}}{100}\right)^{4}-\left(\frac{T_{w 21}}{100}\right)^{4}\right]
$$

- on the vacuum-chamber external surface

$$
\left.\lambda \frac{d T}{d r}\right|_{r_{s 2}}=-\varepsilon_{2} \sigma\left(\frac{T_{w 2}}{100}\right)^{4}+Q_{2}(t)+\alpha_{2}\left(T_{e 2}-T_{w 2}\right) .
$$

The heat losses through the ends of vacuum chamber are set aside; therefore,

$$
\begin{gathered}
\varepsilon_{\mathrm{ad} j}=\frac{1}{1 / \varepsilon_{12}+\left(F_{e} / F_{v}\right)\left(\left(1 / \varepsilon_{21}\right)-1\right)} ; \\
\varphi_{12}=1 ; \quad \varphi_{21}=\frac{F_{e}}{F_{v}} .
\end{gathered}
$$

Here, $\varepsilon_{12}$ and $\varepsilon_{21}, T_{w 12}$ and $T_{w 21}, r_{s 12}$ and $r_{s 21}, \varphi_{12}$ and $\varphi_{21}, F_{e}$ and $F_{v}$ are the degrees of blackness, temperatures, radii, angle coefficients, and the first wall external surface area and the second wall internal surface area, respectively.

Carbon-carbon composite material (CCCM) surface destruction due to interaction between carbon and high-temperature oxidizing flow can be described by the following expression:

$$
\begin{aligned}
\dot{m}_{s} & =\frac{\alpha}{c_{p}} \frac{\sqrt{\left[\left(M_{c} / M\right) \Omega+1\right]^{2}+4 B_{m} \Omega}-\left[\left(M_{c} / M\right) \Omega+1\right]}{2 \Omega} ; \\
\Omega & =\frac{\alpha}{c_{p}} \frac{R T_{w}}{k_{0} P_{w} M_{c}} e^{E /(R T)}
\end{aligned}
$$

where $\alpha / c_{p}$ is the heat-and-mass transfer coefficient taking into account mass transfer due to surface reactions; $k_{0}$ and $E$ are the kinetic constants; $B_{m}$ is the oxidation potential of combustion products; and $M$ and $M_{c}$ are the corresponding molecular weights.

The set of thermal conductivity equations combined with these relationships were solved for nozzle and vacuum chamber simultaneously with boundary-layer equations $[1,2]$. The latter took into account a thermal film in the nozzle area due to jacket cooling of LRE (computational approach is presented in [2]).

\section{RESULTS}

The calculations of nozzle and vacuum chamber thermal states were carried out for the radiant cooling test on oxygen-hydrogen LRE. Figure 6 shows the 


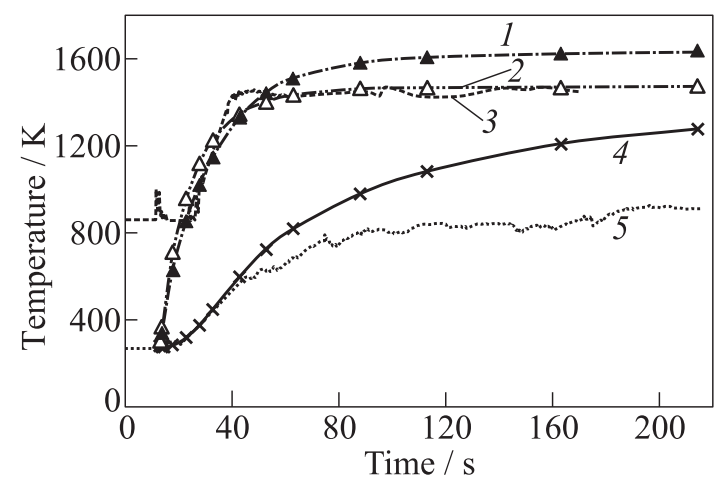

Figure 6 Temperature histories of the external nozzle surface. Thermocouple and IR camera are installed at a distance of $100 \mathrm{~mm}$ from the nozzle root: 1 - calulation without window effect; 2 - calculation with window effect; 3 - IR; 4 - vacuumchamber internal surface temperature; and 5 - thermocouple

calculated time histories of temperature of the nozzle external surface together with the records of the corresponding thermocouples and IR camera.

Typically, the calculated temperature is above that measured by the thermocouple due to the cooling effect of pyrolysis gases at the vacuum-chamber thermoprotected wall (activated at $T>600 \mathrm{~K}$, i.e., after $\sim 40 \mathrm{~s}$ of test).

It is important that the side wall of the vacuum chamber is secured by the eyeglass of large enough diameter to ensure IR diagnostics. The presence of window causes additional heat loss due to radiation. When the mean nozzle

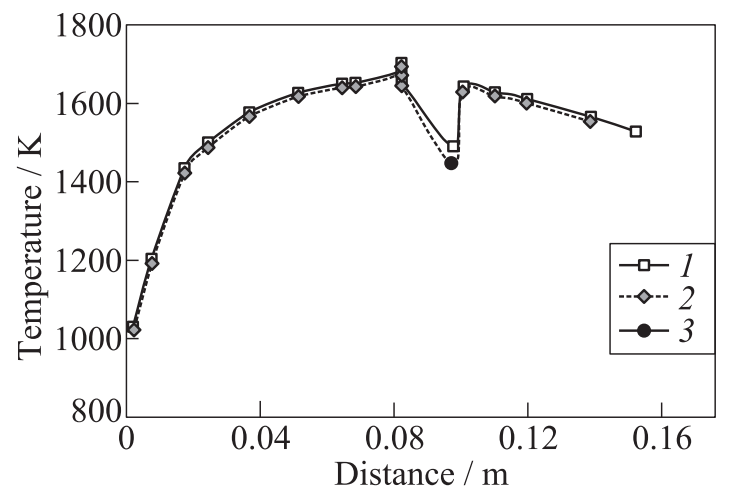

Figure 7 Temperature variation along the nozzle at the end of engine operation: 1 - internal surface temperature; 2 - external surface temperature; and 3 - IR measurements 
temperature is $1650-1700 \mathrm{~K}, 170-220 \mathrm{~K}$ local temperature reduction can be caused by this effect.

The results shown in Fig. 7 demonstrate the methodology under discussion. In this case, the window for IR diagnostics was installed at $85-100 \mathrm{~mm}$ from the nozzle root.

Downward excursion can be seen on the temperature profile in this area. The minimal temperature values agree well with the IR metering results.

\section{CONCLUDING REMARKS}

Thus, the developed software and IR equipment, made on the base of short-wave thermal imager, allow diagnostics of steady-state and rapidly varying thermal fields of high-temperature objects. These tools were successfully used in different technical applications, including distant thermal diagnostics of uncooled LRE nozzles operating at high temperatures (1500-1900 K).

\section{REFERENCES}

1. Keit, F., and W. Bleck 1983. Fundamentals of heat transfer. Moscow: Mir Publ.

2. Volkova, L., N. Volkov, A. Gubertov, and V. Mironov. 2000. Mathematical modeling of heat and mass transfer and thermal protection in engines. Motors 1(7):33-35. 